\title{
THE MULTIPLIER ALGEBRA AND BSE PROPERTY OF THE DIRECT SUM OF BANACH ALGEBRAS
}

\author{
ZEINAB KAMALI ${ }^{\circledR}$ and MAHMOOD LASHKARIZADEH BAMI
}

(Received 13 August 2012; accepted 12 September 2012; first published online 7 February 2013)

\begin{abstract}
The notion of BSE algebras was introduced and first studied by Takahasi and Hatori and later studied by Kaniuth and Ülger. This notion depends strongly on the multiplier algebra $M(\mathcal{A})$ of a commutative Banach algebra $\mathcal{A}$. In this paper we first present a characterisation of the multiplier algebra of the direct sum of two commutative semisimple Banach algebras. Then as an application we show that $\mathcal{A} \oplus \mathcal{B}$ is a BSE algebra if and only if $\mathcal{A}$ and $\mathcal{B}$ are BSE. We also prove that if the algebra $\mathcal{A} \times{ }_{\theta} \mathcal{B}$ with $\theta$-Lau product is a BSE algebra and $\mathcal{B}$ is unital then $\mathcal{B}$ is a BSE algebra. We present some examples which show that the BSE property of $\mathcal{A} \times{ }_{\theta} \mathcal{B}$ does not imply the BSE property of $\mathcal{A}$, even in the case where $\mathcal{B}$ is unital.
\end{abstract}

2010 Mathematics subject classification: primary 46J05; secondary 22D15.

Keywords and phrases: commutative Banach algebra, multiplier algebra, BSE algebra, direct sum.

\section{Introduction}

Let $\mathcal{A}$ be a commutative Banach algebra. Throughout this paper $\Delta(\mathcal{A})$ denotes the set of all nonzero multiplicative linear functionals on $\mathcal{A}$. Then $\Delta(\mathcal{A})$ is a topological space with the Gelfand topology, called the Gelfand spectrum of $\mathcal{A}$.

A bounded continuous function $\sigma$ on $\Delta(\mathcal{A})$ is called a $B S E$ function if there exists a constant $C>0$ such that for every finite number of $\varphi_{1}, \ldots, \varphi_{n}$ in $\Delta(\mathcal{A})$ and the same number of complex numbers $c_{1}, \ldots, c_{n}$, the inequality

$$
\left|\sum_{j=1}^{n} c_{j} \sigma\left(\varphi_{j}\right)\right| \leq C\left\|\sum_{j=1}^{n} c_{j} \varphi_{j}\right\|_{\mathcal{A}^{*}}
$$

holds. The BSE norm of $\sigma,\|\sigma\|_{B S E}$, is defined to be the infimum of all such $C$. The set of all BSE functions is denoted by $C_{B S E}(\Delta(\mathcal{A}))$. Takahasi and Hatori [14] showed that under the norm $\|\cdot\|_{B S E}, C_{B S E}(\Delta(\mathcal{A}))$ is a commutative semisimple Banach algebra.

A bounded linear operator on $\mathcal{A}$ is called a multiplier if it satisfies $x T(y)=T(x y)$ for all $x, y \in \mathcal{A}$. The set $M(\mathcal{A})$ of all multipliers of $\mathcal{A}$ is a closed unital commutative subalgebra of the operator algebra $B(\mathcal{A})$, called the multiplier algebra of $\mathcal{A}$.

(c) 2013 Australian Mathematical Publishing Association Inc. 0004-9727/2013 \$16.00 
For each $T \in M(\mathcal{A})$ there exists a unique continuous function $\widehat{T}$ on $\Delta(\mathcal{A})$ such that $\widehat{T(a)}(\varphi)=\widehat{T}(\varphi) \hat{a}(\varphi)$ for all $a \in \mathcal{A}$ and $\varphi \in \Delta(\mathcal{A})$. See [9] for a proof. Write

$$
\widehat{M(\mathcal{A})}=\{\widehat{T}: T \in M(\mathcal{A})\} .
$$

We say that a commutative Banach algebra $\mathcal{A}$ without order is a BSE algebra (or it is said to have the $B S E$ property) if $\mathcal{A}$ satisfies the condition

$$
C_{B S E}(\Delta(\mathcal{A}))=\widehat{M(\mathcal{A})} \text {. }
$$

Remark 1.1. Let $\mathcal{A}$ be a semisimple Banach algebra and $\Phi: \Delta(\mathcal{A}) \rightarrow \mathbb{C}$ be a continuous function such that $\Phi \cdot \widehat{\mathcal{A}} \subseteq \widehat{\mathcal{A}}$. We call $\Phi$ a multiplier of $\mathcal{A}$. This is another definition of a multiplier of a Banach algebra. In the presence of semisimplicity this definition is equivalent to the above definition, by considering $\Phi=\widehat{T}$; see [9] for more details. Define

$$
\mathcal{M}(\mathcal{A})=\{\Phi: \Delta(\mathcal{A}) \rightarrow \mathbb{C}: \Phi \text { is continuous and } \Phi \widehat{A} \subseteq \widehat{A}\} .
$$

When $\mathcal{A}$ is a semisimple Banach algebra, $\widehat{M(A)}=\mathcal{M}(A)$.

The abbreviation BSE stands for Bochner-Schoenberg-Eberlein and refers to the famous theorem, proved by Bochner and Schoenberg [2, 13] for the additive group of real numbers and by Eberlein [3] for general locally compact abelian groups $G$, saying that, in the above terminology, the group algebra $L^{1}(G)$ is a BSE algebra. (See [11] for a proof.)

The notions of BSE algebra and BSE functions were introduced and studied by Takahasi and Hatori [14, 15] and later by Kaniuth and Ülger [8].

A bounded net $\left(e_{\alpha}\right)_{\alpha}$ in $\mathcal{A}$ is called a bounded approximate identity for $\mathcal{A}$ if it satisfies $\left\|e_{\alpha} a-a\right\| \rightarrow 0$ for all $a \in \mathcal{A}$. A bounded net $\left(e_{\alpha}\right)_{\alpha}$ in $\mathcal{A}$ is called a $\Delta$ weak bounded approximate identity for $\mathcal{A}$ if it satisfies $\varphi\left(e_{\alpha}\right) \rightarrow 1$ (equivalently, $\varphi\left(e_{\alpha} a\right) \rightarrow \varphi(a)$ for every $\left.a \in \mathcal{A}\right)$ for all $\varphi \in \Delta(\mathcal{A})$. Such approximate identities were studied in [6], where the first example was given of a semisimple commutative Banach algebra which has a $\Delta$-weak approximate identity but does not possess a bounded approximate identity. As is shown in [14, Corollary 5], $\mathcal{A}$ has a $\Delta$-weak bounded approximate identity if and only if $\widehat{M(\mathcal{A})} \subseteq C_{B S E}(\Delta(\mathcal{A}))$.

In this paper we first present a characterisation of the multiplier algebra of the direct sum of two semisimple Banach algebras. Then as an application we show that for two semisimple Banach algebras $\mathcal{A}$ and $\mathcal{B}, \mathcal{A} \oplus \mathcal{B}$ is BSE if and only if $\mathcal{A}$ and $\mathcal{B}$ are.

We also prove that if $\mathcal{A}$ and $\mathcal{B}$ are Banach algebras such that $\mathcal{B}$ is unital and $\mathcal{A} \times{ }_{\theta} \mathcal{B}$ is BSE, then $\mathcal{B}$ is a BSE algebra, and we present some examples showing that the BSE property of $\mathcal{A} \times{ }_{\theta} \mathcal{B}$ does not imply that of $\mathcal{A}$.

\section{Direct sum of Banach algebras}

Let $\mathcal{A}$ and $\mathcal{B}$ be two commutative Banach algebras. The direct sum algebra $\mathcal{A} \oplus \mathcal{B}$ of $\mathcal{A}$ and $\mathcal{B}$ is defined as the Cartesian product $\mathcal{A} \times \mathcal{B}$ with the algebra multiplication

$$
\left(a, a^{\prime}\right) \cdot\left(b, b^{\prime}\right)=\left(a a^{\prime}, b b^{\prime}\right) \quad\left(a, a^{\prime} \in \mathcal{A}, b, b^{\prime} \in \mathcal{B}\right),
$$


and with norm

$$
\|(a, b)\|=\|a\|+\|b\| \quad(a \in \mathcal{A}, b \in \mathcal{B}) .
$$

In this section we will give a characterisation of the multiplier algebra of the direct sum algebra $\mathcal{A} \oplus \mathcal{B}, \mathcal{M}(\mathcal{A} \oplus \mathcal{B})$, and then prove that for two semisimple Banach algebras $\mathcal{A}$ and $\mathcal{B}, \mathcal{A} \oplus \mathcal{B}$ is a BSE algebra if and only if $\mathcal{A}$ and $\mathcal{B}$ are BSE algebras. First we need to prove the following lemma.

Lemma 2.1. Let $\mathcal{A}$ and $\mathcal{B}$ be two Banach algebras and

$$
E=\{(\varphi, 0): \varphi \in \Delta(\mathcal{A})\}, \quad F=\{(0, \psi): \psi \in \Delta(\mathcal{B})\}
$$

Then $\Delta(\mathcal{A} \oplus \mathcal{B})=E \cup F$.

Proof. It is obvious that $E \cup F \subseteq \Delta(\mathcal{A} \oplus \mathcal{B})$. For the reverse inclusion suppose that $(\varphi, \psi) \in \Delta(\mathcal{A} \oplus \mathcal{B}) \subseteq \mathcal{A}^{*} \oplus \mathcal{B}^{*}$. Then, for every $a_{1}, a_{2} \in \mathcal{A}$ and $b_{1}, b_{2} \in \mathcal{B}$,

$$
(\varphi, \psi)\left(a_{1} a_{2}, b_{1} b_{2}\right)=(\varphi, \psi)\left(a_{1}, b_{1}\right) \cdot(\varphi, \psi)\left(a_{2}, b_{2}\right)
$$

This means that, for all $a_{1}, a_{2} \in \mathcal{A}$ and $b_{1}, b_{2} \in \mathcal{B}$,

$$
\begin{aligned}
\varphi\left(a_{1} a_{2}\right)+\psi\left(b_{1} b_{2}\right) & =\left(\varphi\left(a_{1}\right)+\psi\left(b_{1}\right)\right) \cdot\left(\varphi\left(a_{2}\right)+\psi\left(b_{2}\right)\right) \\
& =\varphi\left(a_{1}\right) \varphi\left(a_{2}\right)+\varphi\left(a_{1}\right) \psi\left(a_{2}\right)+\psi\left(b_{1}\right) \varphi\left(a_{2}\right)+\psi\left(b_{1}\right) \psi\left(b_{2}\right) .
\end{aligned}
$$

If we take $b_{1}=b_{2}=0$, it follows that $\varphi\left(a_{1} a_{2}\right)=\varphi\left(a_{1}\right) \varphi\left(a_{2}\right)$ for all $a_{1}, a_{2} \in \mathcal{A}$. And similarly, if we take $a_{1}=a_{2}=0$ it follows that $\psi\left(b_{1} b_{2}\right)=\psi\left(b_{1}\right) \psi\left(b_{2}\right)$. Then $\varphi \in \Delta(\mathcal{A}) \cup\{0\}$ and $\psi \in \Delta(\mathcal{B}) \cup\{0\}$. Now if $\varphi=0$, then $(\varphi, \psi)=(0, \psi) \in F$. If $\varphi \neq 0$, then (I) implies that

$$
\varphi\left(a_{1}\right) \psi\left(a_{2}\right)+\psi\left(b_{1}\right) \varphi\left(a_{2}\right)=0,
$$

for all $a_{1}, a_{2} \in \mathcal{A}$ and $b_{1}, b_{2} \in \mathcal{B}$. If we set $a_{1}=0$ and $a_{2}$ such that $\varphi\left(a_{2}\right) \neq 0$, it follows that $\psi\left(b_{1}\right)=0$ for all $b_{1} \in \mathcal{B}$ and then $\psi=0$. This means that $(\varphi, \psi)=(\varphi, 0) \in E$. So $\Delta(\mathcal{A} \oplus \mathcal{B}) \subseteq E \cup F$.

Remark 2.2. Since $E \cup F \subset(\mathcal{A} \oplus \mathcal{B})^{*}=\mathcal{A}^{*} \oplus \mathcal{B}^{*}$, its topology is the one induced from $\mathcal{A}^{*} \oplus \mathcal{B}^{*}$ and is precisely the Gelfand topology of $\Delta(\mathcal{A} \oplus \mathcal{B})$.

Note that Lemma 2.1 implies that $\mathcal{A} \oplus \mathcal{B}$ is semisimple if and only if both $\mathcal{A}$ and $\mathcal{B}$ are semisimple.

Theorem 2.3. Let $\mathcal{A}$ and $\mathcal{B}$ be semisimple Banach algebras. Then

$$
\mathcal{M}(\mathcal{A} \oplus \mathcal{B})=\{(\Phi, \Psi): \Phi \in \mathcal{M}(\mathcal{A}), \Psi \in \mathcal{M}(\mathcal{B})\}
$$

where $(\Phi, \Psi)(\varphi, 0)=\Phi(\varphi)$ and $(\Phi, \Psi)(0, \psi)=\Phi(\psi)$ for all $(\varphi, 0) \in E$ and $(0, \psi) \in F$. 
Proof. Let $\Phi \in \mathcal{M}(\mathcal{A})$ and $\Psi \in \mathcal{M}(\mathcal{B})$. Since $\Phi \widehat{\mathcal{A}} \subseteq \widehat{\mathcal{A}}$ and $\Psi \widehat{\mathcal{B}} \subseteq \widehat{\mathcal{B}}$, then for all $(\varphi, 0) \in E$ and $(a, b) \in \mathcal{A} \oplus \mathcal{B}$, there are elements $a^{\prime} \in \mathcal{A}$ and $b^{\prime} \in \mathcal{B}$ such that

$$
\begin{aligned}
((\Phi, \Psi) \cdot(\widehat{a, b}))(\varphi, 0) & =(\Phi, \Psi)(\varphi, 0) \widehat{(a, b)}(\varphi, 0) \\
& =\Phi(\varphi) \widehat{a}(\varphi)=\widehat{a^{\prime}}(\varphi),
\end{aligned}
$$

for all $\varphi \in \Delta(\mathcal{A})$, and

$$
\begin{aligned}
((\Phi, \Psi) \cdot \widehat{(a, b)})(0, \psi) & =(\Phi, \Psi)(0, \psi) \widehat{(a, b)}(0, \psi) \\
& =\Psi(\psi) \widehat{b}(\psi)=\widehat{b^{\prime}}(\psi),
\end{aligned}
$$

for all $\psi \in \Delta(\mathcal{B})$. Then $((\Phi, \Psi) \cdot \widehat{(a, b)})(\varphi, 0)=\left(\widehat{a^{\prime}, b^{\prime}}\right)(\varphi, 0)$ and $((\Phi, \Psi) \cdot \widehat{(a, b)})(0, \psi)=$ $\left(\widehat{a^{\prime}, b^{\prime}}\right)(0, \psi)$. This implies that

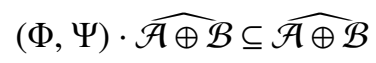

and $(\Phi, \Psi) \in \mathcal{M}(\mathcal{A} \oplus \mathcal{B})$.

Now let $F \in \mathcal{M}(\mathcal{A} \oplus \mathcal{B})$. Define $\Phi(\varphi)=F(\varphi, 0)$ and $\Psi(\psi)=F(0, \psi)$, for all $\varphi \in$ $\Delta(\mathcal{A})$ and $\psi \in \Delta(\mathcal{B})$. So $F=(\Phi, \Psi)$. It is enough to show that $\Phi \in \mathcal{M}(\mathcal{A})$ and $\Psi \in \mathcal{M}(\mathcal{B})$. For all $a \in \mathcal{A}$, there exists $\left(a^{\prime}, b^{\prime}\right) \in \mathcal{A} \oplus \mathcal{B}$ such that

$$
\Phi(\varphi) \widehat{a}(\varphi)=(\Phi, \Psi)(\varphi, 0) \widehat{(a, 0)}(\varphi, 0)=\left(\widehat{a^{\prime}, b^{\prime}}\right)(\varphi, 0)=\widehat{a^{\prime}}(\varphi) .
$$

Then $\Phi \widehat{\mathcal{A}} \subseteq \widehat{\mathcal{A}}$ and $\Phi \in \mathcal{M}(\mathcal{A})$. Similarly, $\Psi \in \mathcal{M}(\mathcal{A})$.

Theorem 2.4. Let $\mathcal{A}$ and $\mathcal{B}$ be two semisimple Banach algebras. Then $\mathcal{A} \oplus \mathcal{B}$ is BSE if and only if $\mathcal{A}$ and $\mathcal{B}$ are $B S E$.

Proof. First suppose that $\mathcal{A}$ and $\mathcal{B}$ are BSE. Then by [14, Corollary 5] $\mathcal{A}$ and $\mathcal{B}$ have $\Delta$-weak bounded approximate identities. Let $\left\{e_{\alpha}\right\}_{\alpha}$ and $\left\{f_{\beta}\right\}_{\beta}$ be $\Delta$-weak bounded approximate identities of $\mathcal{A}$ and $\mathcal{B}$, respectively. Then $\left\{\left(e_{\alpha}, f_{\beta}\right)\right\}_{(\alpha, \beta)}$ is a $\Delta$-weak bounded approximate identity for $\mathcal{A} \oplus \mathcal{B}$. Indeed, for all $\varphi \in \Delta(\mathcal{A})$ and $\psi \in \Delta(\mathcal{B})$,

$$
\lim _{(\alpha, \beta)}(\varphi, 0)\left(e_{\alpha}, f_{\beta}\right)=\lim _{\alpha} \varphi\left(e_{\alpha}\right)=1
$$

and

$$
\lim _{(\alpha, \beta)}(0, \psi)\left(e_{\alpha}, f_{\beta}\right)=\lim _{\beta} \psi\left(f_{\beta}\right)=1,
$$

so that, for all $\Phi \in E \cup F=\Delta(\mathcal{A} \oplus \mathcal{B})$,

$$
\lim _{(\alpha, \beta)} F\left(e_{\alpha}, f_{\beta}\right)=1,
$$

and $\left\{\left(e_{\alpha}, f_{\beta}\right)\right\}_{(\alpha, \beta)}$ is a $\Delta$-weak approximate identity for $\mathcal{A} \oplus \mathcal{B}$. Then by [14, Corollary 5]

$$
\mathcal{M}(\mathcal{A} \oplus \mathcal{B}) \subseteq C_{B S E}(\Delta(\mathcal{A} \oplus \mathcal{B})) .
$$

For the reverse conclusion, let $\sigma \in C_{B S E}(\Delta(\mathcal{A} \oplus \mathcal{B}))$. Then, by [14, Theorem 4(ii)], $\left.\sigma \in C_{b}(\Delta(\mathcal{A} \oplus \mathcal{B})) \cap(\mathcal{A} \oplus \mathcal{B})^{* *}\right|_{\Delta(\mathcal{A} \oplus \mathcal{B})}=\left.C_{b}(\Delta(\mathcal{A} \oplus \mathcal{B})) \cap\left(\mathcal{A}^{* *} \oplus \mathcal{B}^{* *}\right)\right|_{\Delta(\mathcal{A} \oplus \mathcal{B})} . \quad$ Then 
there are $\sigma_{1} \in \mathcal{A}^{* *}$ and $\sigma_{2} \in \mathcal{B}^{* *}$ such that $\left.\left.\sigma_{1}\right|_{\Delta(\mathcal{A})} \in C_{b}(\Delta(\mathcal{A})) \cap \mathcal{A}^{* *}\right|_{\Delta(\mathcal{A})},\left.\sigma_{2}\right|_{\Delta(\mathcal{B})} \in$ $\left.C_{b}(\Delta(\mathcal{B})) \cap \mathcal{B}^{* *}\right|_{\Delta(\mathcal{B})}$ and $\sigma=\left.\left(\sigma_{1}, \sigma_{2}\right)\right|_{\Delta(\mathcal{A} \oplus \mathcal{B})}$. On the other hand, since $\sigma \in$ $C_{B S E}(\Delta(\mathcal{A} \oplus \mathcal{B}))$, there exists $\beta>0$ such that, for every finite number of $c_{1}, \ldots, c_{n} \in \mathbb{C}$ and $\left(\varphi_{1}, \psi_{1}\right), \ldots,\left(\varphi_{n}, \psi_{n}\right) \in \Delta(\mathcal{A} \oplus \mathcal{B})$,

$$
\left|\sum_{i=1}^{n} c_{i} \sigma\left(\varphi_{i}, \psi_{i}\right)\right| \leq \beta\left\|\sum_{i=1}^{n} c_{i}\left(\varphi_{i}, \psi_{i}\right)\right\|_{\mathcal{A}^{*} \oplus \mathcal{B}^{*}} .
$$

In particular, for every $\left(\varphi_{1}, 0\right), \ldots,\left(\varphi_{n}, 0\right) \in E$ and $c_{1}, \ldots, c_{n} \in \mathbb{C}$,

$$
\begin{aligned}
\left|\sum_{i=1}^{n} c_{i} \sigma\left(\varphi_{i}, 0\right)\right| & =\left|\sum_{i=1}^{n} c_{i} \sigma_{1}\left(\varphi_{i}\right)\right| \\
& \leq \beta\left\|\sum_{i=1}^{n} c_{i}\left(\varphi_{i}, 0\right)\right\|_{\mathcal{A}^{*} \oplus \mathcal{B}^{*}} \\
& =\beta \sup \left\{\left|\sum_{i=1}^{n} c_{i}\left(\varphi_{i}, 0\right)(a, b)\right|:\|a\|+\|b\| \leq 1\right\} \\
& \leq \beta \sup \left\{\left|\sum_{i=1}^{n} c_{i} \varphi_{i}(a)\right|:\|a\| \leq 1\right\} \\
& =\beta\left\|\sum_{i=1}^{n} c_{i} \varphi_{i}\right\|_{\mathcal{A}^{*}}
\end{aligned}
$$

This means that $\sigma_{1} \in C_{B S E}(\Delta(\mathcal{A}))$. Now since $\mathcal{A}$ is a semisimple BSE algebra, $\sigma_{1} \in \mathcal{M}(\mathcal{A})$. In a similar way (by considering $\left.\left(0, \psi_{1}\right), \ldots,\left(0, \psi_{n}\right)\right)$ we conclude that $\sigma_{2} \in \mathcal{M}(\mathcal{B})$. So $\sigma=\left(\sigma_{1}, \sigma_{2}\right) \in \mathcal{M}(\mathcal{A} \oplus \mathcal{B})$. Then $C_{B S E}(\Delta(A \oplus B)) \subset \mathcal{M}(\mathcal{A} \oplus \mathcal{B})$ and $\mathcal{A} \oplus \mathcal{B}$ is a BSE algebra.

Now suppose that $A \oplus B$ is BSE and let $\left\{\left(e_{\alpha}, f_{\alpha}\right)\right\}_{\alpha}$ be a $\Delta$-weak bounded approximate identity for $\mathcal{A} \oplus \mathcal{B}$. Then, for all $\varphi \in \Delta(\mathcal{A})$,

$$
\lim _{\alpha} \varphi\left(e_{\alpha}\right)=\lim _{\alpha}(\varphi, 0)\left(e_{\alpha}, f_{\alpha}\right)=1 .
$$

So $\left\{e_{\alpha}\right\}_{\alpha}$ is a $\Delta$-weak bounded approximate identity for $\mathcal{A}$, and similarly $\left\{f_{\alpha}\right\}_{\alpha}$ is a $\Delta$-weak bounded approximate identity for $\mathcal{B}$, and [14, Corollary 5] implies that $\mathcal{M}(\mathcal{A}) \subseteq C_{B S E}(\Delta(\mathcal{A}))$ and $\mathcal{M}(\mathcal{B}) \subseteq C_{B S E}(\Delta(\mathcal{B}))$.

Now let $\sigma_{1} \in C_{B S E}(\Delta(\mathcal{A}))$ and $\sigma_{2} \in C_{B S E}(\Delta(\mathcal{B}))$. Then by [14, Theorem 4(i)] there are nets $\left\{x_{\lambda}\right\}_{\lambda} \subset \mathcal{A}$ and $\left\{y_{\mu}\right\}_{\mu} \in \mathcal{B}$ such that $\lim _{\lambda} \widehat{x_{\lambda}}(\varphi)=\sigma_{1}(\varphi)$ and $\lim _{\mu} \widehat{y_{\mu}}(\psi)=\sigma_{2}(\psi)$, for all $\varphi \in \Delta(\mathcal{A})$ and $\psi \in \Delta(\mathcal{B})$. If we consider the net $\left\{\left(x_{\lambda}, y_{\mu}\right)\right\}_{(\lambda, \mu)} \subset \mathcal{A} \oplus \mathcal{B}$, then

$$
\begin{aligned}
\lim _{(\lambda, \mu)}\left(\widehat{x_{\lambda}, y_{\mu}}\right)(\varphi, 0) & =\lim _{(\lambda, \mu)}(\varphi, 0)\left(x_{\lambda}, y_{\mu}\right)=\lim _{(\lambda, \mu)} \varphi\left(x_{\lambda}\right)+0 \\
& =\lim _{(\lambda, \mu)} \widehat{x_{\lambda}}(\varphi)+0=\sigma_{1}(\varphi)+0 \\
& =\left(\sigma_{1}, \sigma_{2}\right)(\varphi, 0),
\end{aligned}
$$


for all $\varphi \in \Delta(\mathcal{A})$. And similarly, for all $\psi \in \Delta(\mathcal{B})$,

$$
\lim _{(\lambda, \mu)}\left(\widehat{x_{\lambda}, y_{\mu}}\right)(0, \psi)=\left(\sigma_{1}, \sigma_{2}\right)(0, \psi) \text {. }
$$

This means that if we let $\sigma=\left(\sigma_{1}, \sigma_{2}\right)$ then

$$
\lim _{(\lambda, \mu)}\left(\widehat{x_{\lambda}, y_{\mu}}\right)(\Phi)=\sigma(\Phi)
$$

for all $\Phi \in E \cup F=\Delta(\mathcal{A} \oplus \mathcal{B})$. Then $\sigma=\left(\sigma_{1}, \sigma_{2}\right) \in C_{B S E}(\Delta(\mathcal{A} \oplus \mathcal{B}))$. Now since $\mathcal{A} \oplus \mathcal{B}$ is a BSE algebra, $\sigma=\left(\sigma_{1}, \sigma_{2}\right) \in \mathcal{M}(\mathcal{A} \oplus \mathcal{B})$ and, by Theorem 2.3, $\sigma_{1} \in \mathcal{M}(\mathcal{A})$ and $\sigma_{2} \in \mathcal{M}(\mathcal{B})$. So $\mathcal{A}$ and $\mathcal{B}$ are BSE algebras.

Let $G$ be a locally compact abelian group and $M(G)$ the Banach algebra of bounded regular measures on $G$. The set of continuous measures in $M(G)$ is denoted by $M_{c}(G)$. This is a closed ideal in $M(G)$, and

$$
M(G)=M_{d}(G) \oplus M_{c}(G)=l^{1}(G) \oplus M_{c}(G)
$$

When $G$ is not discrete, $M_{c}(G) \neq\{0\}$. It is shown in [14] that $M(G)$ is a BSE algebra if and only if $G$ is discrete. So we have the following result.

Corollary 2.5. For a nondiscrete locally compact abelian group $G, M_{c}(G)$ is not a BSE algebra.

Corollary 2.6. Let $G$ be a locally compact abelian group and $\mu \in M(G)$ be a measure which factors as a product of an invertible measure and an idempotent measure. Then $\mu * L^{1}(G)$ is a BSE algebra.

Proof. Define $T_{\mu}(f)=\mu * f$. Then $T_{\mu}$ is a multiplier of the Banach algebra $L^{1}(G)$. It is shown in [16] that $T\left(L^{1}(G)\right)=\mu * L^{1}(G)$ is closed in $L^{1}(G)$. By [4, Theorem 3.4], since $L^{1}(G)$ is a commutative semisimple amenable Banach algebra, it factors as follows:

$$
L^{1}(G)=T_{\mu}\left(L^{1}(G)\right) \oplus \operatorname{Ker}\left(T_{\mu}\right)=\left(\mu * L^{1}(G)\right) \oplus \operatorname{Ker}\left(T_{\mu}\right)
$$

Since $L^{1}(G)$ is a BSE algebra, by Theorem 2.4, $\mu * L^{1}(G)$ is BSE as well.

\section{3. $\theta$-Lau product of Banach algebras}

The products $\mathcal{A} \times{ }_{\theta} \mathcal{B}$ of Banach algebras $\mathcal{A}$ and $\mathcal{B}$ were first introduced and studied by Lau [10]. The Banach algebra $\mathcal{B}$ inherits some properties of $\mathcal{A} \times{ }_{\theta} \mathcal{B}$. For instance, from [7, Proposition 2.1], for $n \in \mathbb{N}, n$-ideal amenability of $\mathcal{A} \times{ }_{\theta} \mathcal{B}$ implies that of $\mathcal{B}$. In this section we prove that if the $\theta$-Lau product of two Banach algebras $\mathcal{A}$ and $\mathcal{B}$, $\mathcal{A} \times{ }_{\theta} \mathcal{B}$, with $\mathcal{B}$ unital, is BSE, then $\mathcal{B}$ is a BSE algebra. Before that we need to present some definitions and preliminaries. More results on this product can be found in [12]. 
Definition 3.1. Let $\mathcal{A}$ and $\mathcal{B}$ be two commutative Banach algebras for which $\Delta(\mathcal{B}) \neq \emptyset$. Let $\theta \in \Delta(\mathcal{B})$. The $\theta$-Lau product $\mathcal{A} \times{ }_{\theta} \mathcal{B}$ is defined as the Cartesian product $\mathcal{A} \times \mathcal{B}$ with the algebra multiplication

$$
\left(a_{1}, b_{1}\right)\left(a_{2}, b_{2}\right)=\left(a_{1} a_{2}+\theta\left(b_{1}\right) a_{2}+\theta\left(b_{2}\right) a_{1}, b_{1} b_{2}\right)
$$

and norm $\|(a, b)\|=\|a\|+\|b\|$.

Obviously if $\mathcal{B}$ is a unital Banach algebra, then $\mathcal{A} \times{ }_{\theta} \mathcal{B}$ is a unital Banach algebra for any Banach algebra $\mathcal{A}$.

Remark 3.2. The space $\mathcal{A} \times{ }_{\theta} \mathcal{B}$ is a Banach algebra. If we allow $\theta=0$, we obtain the usual direct sum of Banach algebras. If $\mathcal{B}=\mathbb{C}$ and $\theta: \mathbb{C} \rightarrow \mathbb{C}$ is the identity map, then $\mathcal{A} \times{ }_{\theta} \mathbb{C}$ coincides with the unitisation $\mathcal{A}_{e}$ of $\mathcal{A}$.

The dual of the space $\mathcal{A} \times_{\theta} \mathcal{B}$ can be identified with $\mathcal{A}^{*} \oplus \mathcal{B}^{*}$ in the natural way: $(\varphi, \psi)(a, b)=\varphi(a)+\psi(b)$. The dual norm on $\mathcal{A}^{*} \oplus \mathcal{B}^{*}$ is the maximum norm $\|(\varphi, \psi)\|=\max \{\|\varphi\|,\|\psi\|\}$. On $\mathcal{A}^{*} \oplus \mathcal{B}^{*}$, the weak* topology coincides with the product of the weak* topologies of $\mathcal{A}^{*}$ and $\mathcal{B}^{*}$. The following theorem, which is proved in [12], identifies the Gelfand spectrum $\Delta\left(\mathcal{A} \times{ }_{\theta} \mathcal{B}\right)$ of $\mathcal{A} \times_{\theta} \mathcal{B}$.

Theorem 3.3. Let $\mathcal{A}$ and $\mathcal{B}$ be Banach algebras with $\Delta(\mathcal{B}) \neq \emptyset$. Let $\theta \in \Delta(\mathcal{B})$ and

$$
E=\{(\varphi, \theta): \varphi \in \Delta(\mathcal{A})\}, \quad F=\{(0, \psi): \psi \in \Delta(\mathcal{B})\} .
$$

Set $E=\emptyset$ if $\Delta(A)=\emptyset$. Then $\Delta\left(A \times_{\theta} B\right)=E \cup F$.

Note that the topology on $E \cup F=\Delta\left(\mathcal{A} \times{ }_{\theta} \mathcal{B}\right)$ is the weak* topology induced from $\left(\mathcal{A} \times{ }_{\theta} \mathcal{B}\right)^{*}=\mathcal{A}^{*} \oplus \mathcal{B}^{*}$ and it is precisely the Gelfand topology on $\Delta\left(\mathcal{A} \times{ }_{\theta} \mathcal{B}\right)$.

Theorem 3.4. Let $\mathcal{A}$ and $\mathcal{B}$ be two commutative Banach algebras. Suppose that $\mathcal{A} \times{ }_{\theta} \mathcal{B}$ is a BSE algebra. Then $\mathcal{B}$ is a BSE algebra.

Proof. Let $\sigma \in C_{B S E}(\Delta(\mathcal{B}))$. Then by [14, Theorem 4(i)], there exists a bounded net $\left\{y_{\lambda}\right\}_{\lambda} \subset \mathcal{B}$ such that $\lim _{\lambda} \widehat{y_{\lambda}}(\psi)=\sigma(\psi)$, for all $\psi \in \Delta(\mathcal{B})$. If we consider the bounded net $\left\{\left(0, y_{\lambda}\right)\right\}_{\lambda} \subset \mathcal{A} \times_{\theta} \mathcal{B}$,

$$
\begin{aligned}
\lim _{\lambda}\left(\widehat{0, y_{\lambda}}\right)(0, \psi) & =\lim _{\lambda}(0, \psi)\left(0, y_{\lambda}\right)=\lim _{\lambda} 0+\widehat{y_{\lambda}}(\psi) \\
& =\sigma(\psi)=(0, \sigma)(0, \psi),
\end{aligned}
$$

for all $(0, \psi) \in E$. Also, for all $(\varphi, \theta) \in F$,

$$
\begin{aligned}
\lim _{\lambda}\left(\widehat{0, y_{\lambda}}\right)(\varphi, \theta) & =\lim _{\lambda}(\varphi, \theta)\left(0, y_{\lambda}\right)=\lim _{\lambda} \widehat{y_{\lambda}}(\theta) \\
& =\sigma(\theta)=(0, \sigma)(\varphi, \theta) .
\end{aligned}
$$

Consequently, for all $\Phi \in E \cup F=\Delta\left(\mathcal{A} \times{ }_{\theta} \mathcal{B}\right)$,

$$
\lim _{\lambda}\left(\widehat{0, y_{\lambda}}\right)(\Phi)=(0, \sigma)(\Phi) \text {. }
$$


Then $(0, \sigma) \in C_{B S E}\left(\Delta\left(\mathcal{A} \times_{\theta} \mathcal{B}\right)\right)$ and since $\mathcal{A} \times_{\theta} \mathcal{B}$ is BSE, $(0, \sigma) \in\left(\widehat{\mathcal{A} \times{ }_{\theta}} \mathcal{B}\right)$. So there exists $(a, b) \in \mathcal{A} \times{ }_{\theta} \mathcal{B}$ such that $\widehat{(a, b)}=(0, \sigma)$. Then, for all $\psi \in \Delta(\mathcal{B})$,

$$
\sigma(\psi)=(0, \sigma)(0, \psi)=\widehat{(a, b)}(0, \psi)=(0, \psi)(a, b)=\widehat{b}(\psi) .
$$

This means that $\sigma \in \widehat{\mathcal{B}}$ and since $\mathcal{B}$ is unital, $\mathcal{B}$ is a BSE algebra.

The following examples show that if $\mathcal{A} \times{ }_{\theta} \mathcal{B}$, for $\mathcal{B}$ unital, is BSE we cannot conclude that $\mathcal{A}$ is BSE in general. Before that we need to present a result proved by Kaniuth and Ülger, [8, Theorem 4.8].

Theorem 3.5. Let $\mathcal{A}$ be a nonunital commutative Banach algebra. Then the unitisation $\mathcal{A}_{e}$ of $\mathcal{A}$ is a BSE algebra if and only if

$$
C_{B S E}(\Delta(\mathcal{A})) \cap C_{0}(\Delta(\mathcal{A}))=\widehat{\mathcal{A}}
$$

EXAMPLE 3.6.

(1) Let $G$ be a second countable noncompact locally compact group whose regular representation is not completely reducible and $A(G)$ be the Fourier algebra of $G$. Then $A(G) \neq B(G) \cap C_{0}(G)$ (see $[1,5,8]$ ). Thus if in addition $G$ is amenable, then $A(G)$ is a BSE algebra [8], but $A(G)_{e}=A(G) \times_{\theta} \mathbb{C}$, such that $\theta: \mathbb{C} \rightarrow \mathbb{C}$ is the identity map, is not BSE, by Theorem 3.5

(2) Let $l^{1}(\mathbb{N})$ be the semigroup algebra of the additive semigroup of natural numbers. Then $l^{1}(\mathbb{N})$ is not a BSE algebra [14]. However, the semigroup algebra $l^{1}(\mathbb{N} \cup\{0\})=l^{1}(\mathbb{N})_{e}=l^{1}(\mathbb{N}) \times_{\theta} \mathbb{C}$, such that $\theta: \mathbb{C} \rightarrow \mathbb{C}$ is the identity map, is a BSE algebra by [15, Theorem 6].

\section{Acknowledgements}

The authors would like to thank the Center of Excellence for Mathematics and the office of Graduate Studies of the University of Isfahan for their financial support.

\section{References}

[1] L. Baggett and K. Taylor, 'A sufficient condition for the complete reducibility of the regular representation', J. Funct. Anal. 34 (1979), 250-265.

[2] S. Bochner, 'A theorem on Fourier-Stieltjes integrals', Bull. Amer. Math. Soc. 40 (1934), 271-276.

[3] W. F. Eberlein, 'Characterizations of Fourier-Stieltjes transforms', Duke. Math. J. 22 (1955), $465-468$.

[4] A. Fattahi, 'Multiplier on character amenable Banach algebras', Int. Math. Forum 42 (2010), 2085-2091.

[5] A. Figà-Talamanca, 'Positive definite functions which vanish at infinity', Pacific J. Math. 69 (1977), 355-363.

[6] C. A. Jones and C. D. Lahr, 'Weak and norm approximate identities are different', Pacific J. Math. 72 (1977), 99-104.

[7] Z. Kamali and M. Nemati, 'On $n$-ideal amenability of certain Banach algebras', Bull. Aust. Math. Soc. 86 (2012), 90-99. 
[8] E. Kaniuth and A. Ülger, 'The Bochner-Schoenberg-Eberlein property for commutative Banach algebras, especially Fourier and Fourier-Stieltjes algebras', Trans. Amer. Math. Soc. 362 (2010), 4331-4356.

[9] R. Larsen, An Introduction to the Theory of Multipliers (Springer, New York, 1971).

[10] A. T.-M. Lau, 'Analysis on a class of Banach algebras with applications to harmonic analysis on locally compact groups and semigroups', Fund. Math. 118 (1983), 161-175.

[11] W. Rudin, Fourier Analysis on Groups (Wiley Interscience, New York, 1984).

[12] M. Sangani Monfared, 'On certain products of Banach algebras with applications to harmonic analysis', Studia Math. 178 (2007), 277-294.

[13] I. J. Schoenberg, 'A remark on the preceeding note by Bochner', Bull. Amer. Math. Soc. 40 (1934), 277-278.

[14] S.-E. Takahasi and O. Hatori, 'Commutative Banach algebras which satisfy a BochnerSchoenberg-Eberlin-type theorem’, Proc. Amer. Math. Soc. 110 (1990), 149-158.

[15] S.-E. Takahasi and O. Hatori, 'Commutative Banach algebras and BSE-inequalities', Math. Japonica 37 (1992), 47-52.

[16] Y. Zaime, 'Opérateurs de convolution d'image fermé et unité approchés', Bull. Sci. Math $2 e$ Sèr. 99 (1975), 65-74.

\author{
ZEINAB KAMALI, Department of Mathematics, \\ Faculty of Science, University of Isfahan, Isfahan, Iran \\ e-mail: ze.kamali@sci.ui.ac.ir
}

MAHMOOD LASHKARIZADEH BAMI, Department of Mathematics, Faculty of Science, University of Isfahan, Isfahan, Iran

e-mail: lashkari@sci.ui.ac.ir 